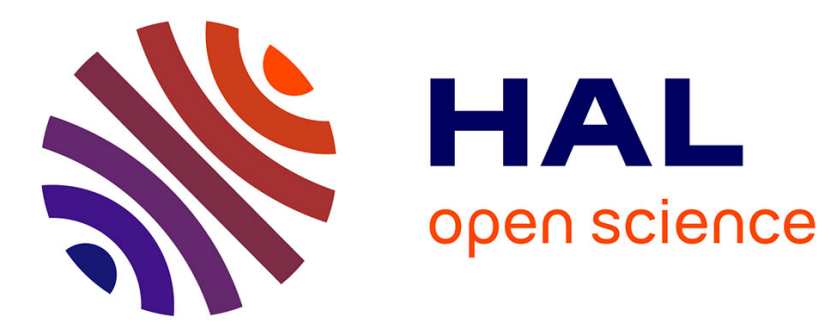

\title{
Set estimation, computation of volumes and data safety
}

\author{
Isabelle Braems, Luc Jaulin, Michel Kieffer, Eric Walter
}

\section{To cite this version:}

Isabelle Braems, Luc Jaulin, Michel Kieffer, Eric Walter. Set estimation, computation of volumes and data safety. SCAN-Interval 2000, Sep 2000, Karlsrhue, Germany. pp.267-280. hal-00845041

\section{HAL Id: hal-00845041 \\ https://hal.science/hal-00845041}

Submitted on 16 Jul 2013

HAL is a multi-disciplinary open access archive for the deposit and dissemination of scientific research documents, whether they are published or not. The documents may come from teaching and research institutions in France or abroad, or from public or private research centers.
L'archive ouverte pluridisciplinaire HAL, est destinée au dépôt et à la diffusion de documents scientifiques de niveau recherche, publiés ou non, émanant des établissements d'enseignement et de recherche français ou étrangers, des laboratoires publics ou privés. 


\title{
SET ESTIMATION, COMPUTATION OF VOLUMES AND DATA SAFETY
}

\author{
Isabelle Braems ${ }^{1}$ \\ Luc Jaulin ${ }^{2}$ \\ Michel Kieffer ${ }^{1}$ \\ and Éric Walter ${ }^{1}$ \\ ${ }^{1}$ Laboratoire des Signaux et Systèmes, CNRS - Supélec - Université Paris-Sud \\ Plateau de Moulon, 91192 Gif-sur-Yvette, France \\ \{braems,kieffer,walter\}@lss.supelec.fr \\ ${ }^{2}$ Laboratoire d'Ingénierie des Systèmes Automatisés, Université d'Angers, France \\ jaulin@sciences.univ-angers.fr
}

Keywords: bounded errors, outlier, parameter identification, set estimation.

Abstract This paper proposes a new method for bracketing a set $\mathbb{S}$ defined by nonlinear inequalities between an inner set $\mathbb{S}^{-}$and an outer set $\mathbb{S}^{+}$. Contrary to existing approaches for which $\mathbb{S}^{-}$and $\mathbb{S}^{+}$are unions of boxes, these two sets are defined as unions of polytopes. This characterization makes it possible to describe $\mathbb{S}$ and compute its volume in a more accurate way than with classical methods. The resulting approach is used to quantify the influence of a given interval datum in parameter estimation, when the feasible set for the parameters is defined as the set of all parameter vectors consistent with all interval data. In order to detect potential outliers, we characterize the influence of any given datum on this set by its safety defined as the ratio between the volumes of the feasible sets computed with and without this datum. This problem amounts to computing volumes of sets as accurately as possible.

\section{BOUNDED ERROR ESTIMATION}

The problem to be considered is the critical analysis of measurements used to estimate parameters under the assumption of bounded errors. The $m$-vector $\mathbf{x}$ to be estimated parametrizes a nonlinear mathematical model structure $\mathbf{f}$ that has been chosen to describe the observed behavior of some physical system. Its $m$ components $x_{i}$ are assumed to be constant for the sake of simplicity. A prior domain $\mathbb{X} \subseteq \mathbb{R}^{m}$ for $\mathbf{x}$ is provided by the hypotheses associated with the model. From measurements per- 
formed on the physical system we collect a vector of experimental noisy data $\mathbf{y}^{\#} \in \mathbb{R}^{n}$, that is only a realization of the actual system output $\mathbf{y}$ to be compared to $\mathbf{f}(\mathbf{x})$. In the bounded-error context, it is assumed that the experimental procedure supplies a bound on the amplitude of the additive noise corrupting each datum $y_{i}^{\#}$ and thus defines the support for the variable $y_{i}$ as an uncertainty interval $\left[y_{i}\right]=\left[y_{i}^{-} ; y_{i}^{+}\right]$. From the interval vector $[\mathbf{y}]=\left(\left[y_{1}\right], \ldots,\left[y_{n}\right]\right)^{\mathrm{T}} \mathbf{x}$ is then estimated by characterizing the set $\mathbb{S}$ of all feasible parameters, i.e.

$$
\mathbb{S}=\{\mathbf{x} \in \mathbb{X} \mid \exists \mathbf{y} \in[\mathbf{y}] \mathbf{y}=\mathbf{f}(\mathbf{x})\}=\mathbb{X} \cap \mathbf{f}^{-1}([\mathbf{y}]),
$$

see Figure 1. Characterizing $\mathbb{S}$ is a set-inversion problem. The corresponding direct problem is a filtering problem defined, in the same bounded-error context, as the characterization of $\mathbb{Y}^{*}$ such that $\mathbb{Y}^{*}=$ $\mathbb{Y} \cap \mathbf{f}(\mathbb{X})$ (see Figure 1). $\mathbb{S}$ defines the uncertainty region for $\mathbf{x}$, and its

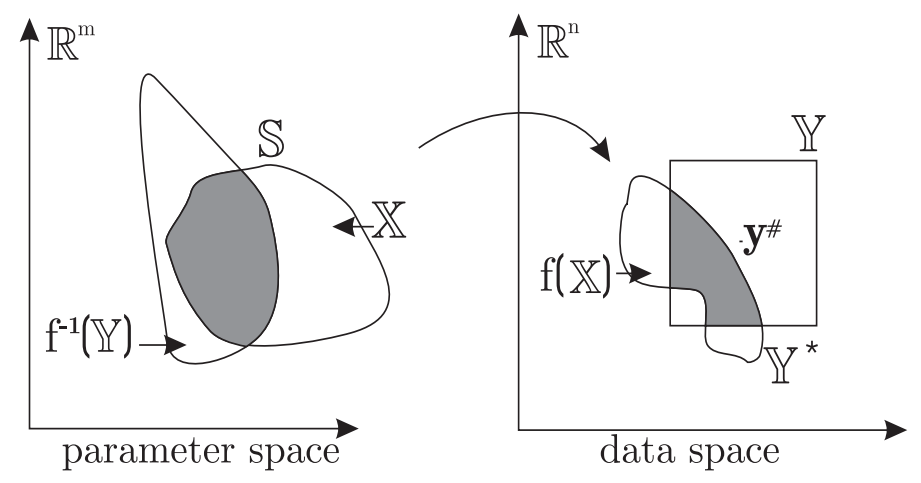

Figure 1 Set inversion (computation of $\mathbb{S}=\mathbb{X} \cap \mathbf{f}^{-1}([\mathbf{y}])$ ) and filtering (computation of $\left.\mathbb{Y}^{*}=\mathbb{Y} \cap \mathbf{f}(\mathbb{X})\right)$.

projection onto each axis $i$ provides a feasible interval for $x_{i}$. Its size is thus a global indicator of the accuracy with which the parameters are estimated. As $\mathbb{S}$ depends on the experiments through $[\mathbf{y}]$, it is possible to evaluate the pertinence of the experimental measurements a posteriori by characterizing $\mathbb{S}$. Define $\mathbf{y}^{*}$ as the $n$-data vector that would be obtained if the measurements were noise-free. In practice, it may happen that $y_{i}^{*}$ does not belong to $\left[y_{i}\right]$, for instance in case of a sensor failure, or if the model is not rich enough to describe the system properly. Such a datum is called an outlier. The presence of outliers may dramatically change the solution set $\mathbb{S}$, to the point that $\mathbb{S}$ may even become empty. Detecting outliers thus turns out to be a critical issue. In the following, we propose to associate to each datum a value called safety based on its 
impact on the volume of the feasible set that will help us to characterize the influence of each datum and detect outliers. Obviously, the quality of the detection will depend on the accuracy of the description of $\mathbb{S}$ and of the computation of its volume $\operatorname{Vol}(\mathbb{S})$. Classical subbdivision algorithms such as SIVIA [7], [10] compute a guaranteed estimate of $\mathbb{S}$. It is then easy to obtain an estimate of $\operatorname{Vol}(\mathbb{S})$. We shall show that it is possible to improve the accuracy of the description of $\mathbb{S}$, and that of its volume, without any significant computing burden, by computing on polytopes. The new algorithm SIVIA1 evaluates with more accuracy the set $\mathbb{S}$ and in the same time computes its volume more efficiently. Section 2 recalls the classical algorithm SIVIA used to evaluate $\mathbb{S}$ and its limitations, and focuses on the concept of reliable linearization of a system. SIVIA1, a new algorithm implementing this concept in a SIVIA structure is described in Section 3. The application to the evaluation of the safety of a datum is then illustrated in Section 4 .

\section{SET ESTIMATION USING IA}

Since $\mathbb{S}$ is the reciprocal image of a box by a nonlinear function, it usually cannot be computed exactly. Interval Analysis (IA) however makes it possible to bracket it between two sets $\mathbb{S}^{-}$and $\mathbb{S}^{+}$such that

$$
\mathbb{S}^{-} \subset \mathbb{S} \subset \mathbb{S}^{+}
$$

As will be shown, the choice of the structure of $\mathbb{S}^{-}$and $\mathbb{S}^{+}$determines the accuracy of the description. The classical approach is to consider $\mathbb{S}^{-}$and $\mathbb{S}^{+}$as unions of non-overlapping boxes, as in SIVIA. SIVIA partitions the prior domain $\mathbb{X}$ into non-overlapping boxes $[\mathbf{x}]$ that are tested and put into one of the following lists:

- the list $\mathcal{L}_{F}$ of the boxes that have been proved feasible,

- the list $\mathcal{L}_{R}$ of the boxes that have been proved unfeasible (optional).

- the list $\mathcal{L}_{U}$ of undetermined boxes that are bisected before undergoing test unless their width is less than $\varepsilon$.

Eventually, SIVIA provides $\mathcal{L}_{F}$ and $\mathcal{L}_{U}$ such that $\mathcal{L}_{F} \subset \mathbb{S} \subset\left(\mathcal{L}_{F} \cup\right.$ $\left.\mathcal{L}_{U}\right)$. The value chosen for $\varepsilon$ determines the accuracy of the enclosure of the boundary of $\mathbb{S}$ in $\mathcal{L}_{U}$. Of course, $\operatorname{Vol}(\mathbb{S}) \in\left[\operatorname{Vol}\left(\mathbb{S}^{-}\right) ; \operatorname{Vol}\left(\mathbb{S}^{+}\right)\right]=$ $\left[\operatorname{Vol}\left(\mathcal{L}_{F}\right) ; \operatorname{Vol}\left(\mathcal{L}_{F}\right)+\operatorname{Vol}\left(\mathcal{L}_{U}\right)\right]$. The accuracy of the computation of $\operatorname{Vol}(\mathbb{S})$ thus depends on the size of $\mathcal{L}_{U}$, and we shall quantify it by

$$
\eta=\frac{1}{\operatorname{Vol}\left(\mathcal{L}_{U}\right)}
$$


As $\varepsilon$ decreases, $\eta$ increases and the enclosure becomes more accurate, at the cost, of course, of a potentially significant increase in the computing burden. To avoid combinatorial problems, we propose hereafter a new strategy to get a better enclosure of $\operatorname{Vol}(\mathbb{S})$, by reducing the volume of $\Delta \mathbb{S}$, and changing the nature of the objects stored in the lists.

Example 1 Consider the following bidimensional system:

$$
\begin{aligned}
\mathbf{x} & \in \mathbb{X}=[0 ; 5] \times[0 ; 5] ; \\
\mathbf{f} & : \mathbb{R}^{2} \rightarrow \mathbb{R}^{3} \\
\mathbf{x} & \rightarrow\left(\begin{array}{l}
x_{2}-2 x_{1} \\
x_{2}-6 / x_{1} \\
x_{2}-0.5 \sqrt{x_{1}}
\end{array}\right) \text { with } \\
{[\mathbf{y}] } & =([-1 ; 1] ;[-2 ; 2] ;[-2 ; 2])^{T}
\end{aligned}
$$

The sets $\mathbb{S}^{-}$and $\mathbb{S}^{+}$computed by SIVIA are depicted on Figure 2. Decreasing $\varepsilon$ increases the number of bisections, and thus the computational burden. In higher dimensions the situation is obviously worse.

3

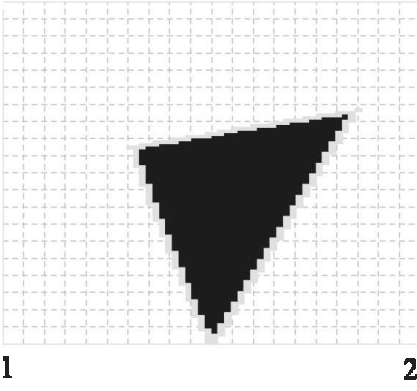

3

2

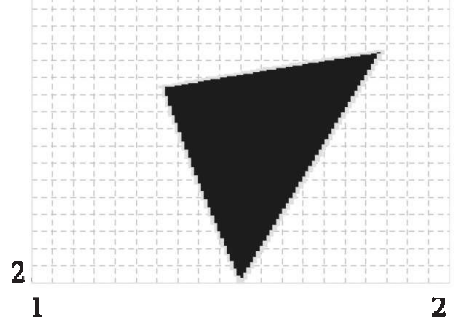

Figure 2 $\mathbb{S}$ of Example 1 is bracketed between $\mathbb{S}^{-}$(black) and $\mathbb{S}^{+}$(light gray) computed by SIVIA. Left: $\varepsilon=0.01$, with 638 bissections, in $0.394 \mathrm{sec}, \operatorname{Vol}\left(\mathcal{L}_{U}\right)=$ 3.4.10 ${ }^{-3}$. Right: $\varepsilon=0.005$, with 1269 bissections, in $0.771 \mathrm{sec}, \operatorname{Vol}\left(\mathcal{L}_{U}\right)=1.2 .10^{-3}$.

\subsection{RELIABLE LINEARIZATION}

Since the computing time increases exponentially when SIVIA bisects the boxes for higher dimensional problems, we aim at reducing the size of the undetermined boxes $[\mathbf{x}]$ stored in $\mathcal{L}_{U}$ without bisecting them anymore. The problem to be considered can be defined as follows:

Assume that a set $\mathbb{S}$ has been enclosed between two unions of boxes $\mathbb{S}^{-}$and $\mathbb{S}^{+}$such that $\mathbb{S}^{-} \subset \mathbb{S} \subset \mathbb{S}^{+}$, with $\mathbb{S}^{+}=\mathbb{S}^{-} \cup \Delta \mathbb{S}$. Can the size of 
$\Delta \mathbb{S}$ be reduced without any bisection, under the condition that the boxes in $\Delta \mathbb{S}$ are small? Let $[\mathbf{x}]$ be a box of $\Delta \mathbb{S}$, and $\mathbb{S}_{[\mathbf{x}]}=\mathbb{S} \cap[\mathbf{x}]$. Reducing the size of $\Delta \mathbb{S}$ means finding a more accurate enclosure $\left(\mathcal{X}^{-} ; \mathcal{X}^{+}\right)$of $\mathbb{S}_{[\mathbf{x}]}$ in $[\mathbf{x}]$, such that $\mathcal{X}^{-} \subset \mathbb{S}_{[\mathbf{x}]} \subset \mathcal{X}^{+} \subset[\mathbf{x}]$. When only $\mathcal{X}^{+}$is to be found, under the constraint that $\mathcal{X}^{+}$is a box, some algorithms already exist. As they transform a pessimistic box enclosure $[\mathbf{x}]$ into a more accurate one $\mathcal{X}^{+}=\left[\mathbf{x}^{+}\right]$, they are called contractors in the literature. See for instance interval constraint propagation algorithms (ICP [4], [6]). Unfortunately, if the boundary $\partial \mathbb{S}$ of $\mathbb{S}$ is far from parallel to axes of the parameter space, accuracy increase will require many bisections, due to the wrapping effect. Moreover, this approach only computes an external approximation and can thus not improve the accuracy of $\mathbb{S}^{-}$. In order to take the gradient of $\partial \mathbb{S}$ into account in the reduction procedure, we propose to linearize the functions $f_{i}(\mathbf{x})$ on each box $[\mathbf{x}]$ of $\Delta \mathbb{S}$. The next three paragraphs explain how to compute $\mathcal{X}^{+}$and $\mathcal{X}^{-}$for the set $\mathbb{S}$.

Reliable Linearization. The case under study is defined by (1.1). Contrary to the objects treated by the interval Newton method [1] [9] $\mathbb{S}$ does not have an empty volume and is a priori bounded by $2 n$ constraints with $2 n \neq m$. We shall define an external enclosure for $\mathbb{S}$, by incorporating the nonlinearities discarded by the linearized system in the uncertainties $\left[y_{i}\right]$. We bracket each $f_{i}(\mathbf{x})$ on $[\mathbf{x}]$ between two parallel hyperplanes, in order to write

$$
\mathbf{A} \cdot \mathbf{x}+\mathbf{b}^{-} \leq \mathbf{f}(\mathbf{x}) \leq \mathrm{A} \cdot \mathbf{x}+\mathrm{b}^{+} .
$$

To compute such hyperplanes, we use the mean value theorem extended to interval functions:

$$
\forall \mathbf{x} \in[\mathbf{x}], \mathbf{f}(\mathbf{x}) \in \mathbf{f}\left(\mathbf{x}_{0}\right)+\left[\frac{d \mathbf{f}}{d \mathbf{x}}\right]([\mathbf{x}])\left(\mathbf{x}-\mathbf{x}_{0}\right), \text { with } \mathbf{x}_{0}=\operatorname{center}([\mathbf{x}]),
$$

where $\left[\frac{d \mathbf{f}}{d \mathbf{x}}\right]([\mathbf{x}])$ is an inclusion function for the Jacobian matrix $\left[\frac{d \mathbf{f}}{d \mathbf{x}}(\mathbf{x})\right]$. We thus have $\mathbf{f}(\mathbf{x}) \in \mathbf{A} . \mathbf{x}+[\mathbf{b}]$ with

$$
\begin{gathered}
\mathbf{A}=\left(\frac{d \mathbf{f}}{d \mathbf{x}}\left(\mathbf{x}_{0}\right)\right) \\
{[\mathbf{b}]=\mathbf{f}\left(\mathbf{x}_{0}\right)-\frac{d \mathbf{f}}{d \mathbf{x}}\left(\mathbf{x}_{0}\right) \cdot \mathbf{x}_{0}+\left(\left[\frac{d \mathbf{f}}{d \mathbf{x}}\right]([\mathbf{x}])-\frac{d \mathbf{f}}{d \mathbf{x}}\left(\mathbf{x}_{0}\right)\right)\left([\mathbf{x}]-\mathbf{x}_{0}\right) .}
\end{gathered}
$$

Note that

$$
\frac{w([\mathbf{b}])}{w([\mathbf{x}])}=\frac{w\left(\left(\left[\frac{d \mathbf{f}}{d \mathbf{x}}\right]([\mathbf{x}])-\frac{d \mathbf{f}}{d \mathbf{x}}\left(\mathbf{x}_{0}\right)\right)\left([\mathbf{x}]-\mathbf{x}_{0}\right)\right)}{w([\mathbf{x}])}
$$


so $w([\mathbf{b}]) / w([\mathbf{x}]) \rightarrow 0$ when $w([\mathbf{x}]) \rightarrow 0$ if $\left[\frac{d \mathbf{f}}{d \mathbf{x}}\right]$ converges, which will be assumed. The accuracy of the enclosure thus grows as $[\mathbf{x}]$ converges to a point $\mathbf{x}$.

External Linearization. We start by computing the element $\mathcal{X}^{+}$. As we want to bracket the element $\mathbb{S}_{[\mathbf{x}]}$ of the solution set, we can write

$$
\begin{aligned}
\mathbf{f}(\mathbf{x}) \in[\mathbf{y}] & \Rightarrow \exists \mathbf{b} \in[\mathbf{b}], \exists \mathbf{y} \in[\mathbf{y}] \mid \mathbf{A} \cdot \mathbf{x}+\mathbf{b}=\mathbf{y} \\
& \Leftrightarrow \mathbf{A} \cdot \mathbf{x} \in[\mathbf{y}]-[\mathbf{b}]=\left[\mathbf{y}^{-}-\mathbf{b}^{+}, \mathbf{y}^{+}-\mathbf{b}^{-}\right],
\end{aligned}
$$

Let $\left[\mathbf{c}_{\mathbf{e}}\right]=\left[\mathbf{y}^{-}-\mathbf{b}^{+}, \mathbf{y}^{+}-\mathbf{b}^{-}\right]$and $\left\langle\mathbf{x}^{+}\right\rangle$be the set solution of $\left\{\begin{array}{l}\mathbf{A} \cdot \mathbf{x} \in\left[\mathbf{c}_{\mathbf{e}}\right] \\ \mathbf{x} \in[\mathbf{x}]\end{array}\right.$. As $\left\langle\mathbf{x}^{+}\right\rangle$is a finite intersection of closed half-spaces, it is a compact convex polyhedron, i.e. a polytope (see Figure 3, left), which contains each $[\mathbf{x}]$ belonging to $\mathbb{S}_{[\mathbf{x}]}$.

Remark 1 Note that the smallest box $\left[\mathbf{x}_{m}\right]$ containing the solutions of the linear system can be computed using linear programming techniques, in order to get the reduced box $[\mathbf{q}]=[\mathbf{x}] \cap\left[\mathbf{x}_{m}\right]$ (see Figure 3, left). The algorithm providing $[\mathbf{q}]$ is then a contractor.

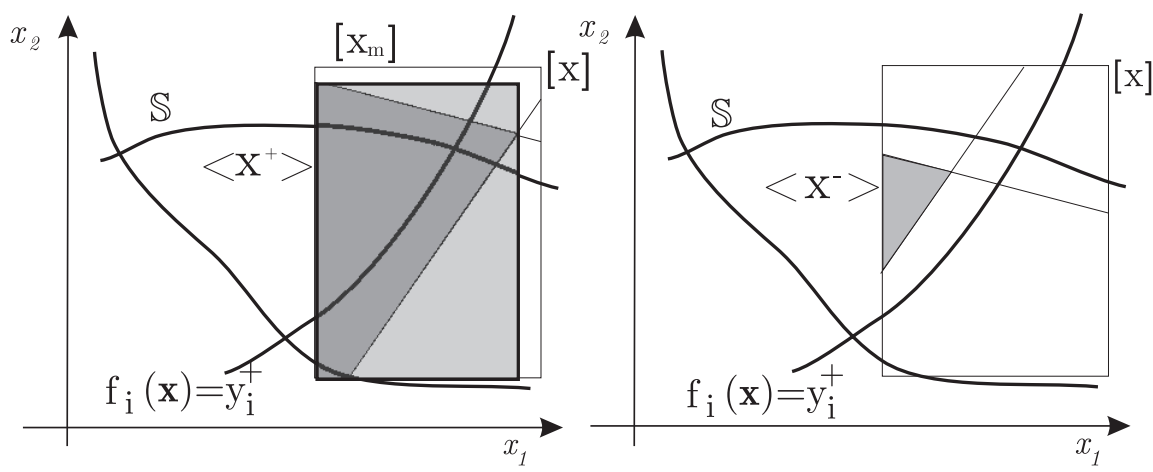

Figure 3 Left: objects obtained after applying two reduction techniques on the box $[\mathbf{x}]:\left[\mathbf{x}_{m}\right]$ is the reduced box obtained by interval constraint propagation; in dark grey, the set $\left\langle\mathbf{x}^{+}\right\rangle$enclosed by the hyperplanes computed by external linearization. Right: Calculus of the internal linearization $\left\langle\mathbf{x}^{-}\right\rangle$of $\mathbb{S}$ on the box $[\mathbf{x}]$.

Internal Linearization. The computation of an internal enclosure $\mathcal{X}^{-}$for $\mathbb{S}$ on the box $[\mathbf{x}]$ follows the same process. Consider any $\mathbf{x} \in[\mathbf{x}]$ such that A.x $\in\left[\mathbf{c}_{\mathbf{i}}\right]$ where

$$
\left[\mathbf{c}_{\mathbf{i}}\right]=\left[\mathbf{y}^{-}-\mathbf{b}^{-} ; \mathbf{y}^{+}-\mathbf{b}^{+}\right] .
$$


Then

$$
\begin{aligned}
\mathbf{A} . \mathbf{x} & \geq \mathbf{y}^{-}-\mathbf{b}^{-} \text {and } \mathbf{A} \cdot \mathbf{x} \leq \mathbf{y}^{+}-\mathbf{b}^{+} \\
& \Leftrightarrow\left[\mathbf{A} \cdot \mathbf{x}+\mathbf{b}^{-} ; \mathbf{A} \cdot \mathbf{x}+\mathbf{b}^{+}\right] \subset\left[\mathbf{y}^{-} ; \mathbf{y}^{+}\right] \\
& \Rightarrow \mathbf{f}(\mathbf{x}) \in[\mathbf{y}]
\end{aligned}
$$

where $\mathbf{A}$ and $[\mathbf{b}]$ are defined by (1.4) and (1.5). We have then proven that any $\mathbf{x}$ satisfying $\left\{\begin{array}{l}\mathbf{x} \in[\mathbf{x}] \\ \mathbf{A} . \mathbf{x} \in\left[\mathbf{c}_{\mathbf{i}}\right]\end{array}\right.$ is in $\mathbb{S}_{[\mathbf{x}]}$. The set defined by (3) is once again a polytope, noted $\left\langle\mathbf{x}^{-}\right\rangle$, included in $\left\langle\mathbf{x}^{+}\right\rangle$(see Figure 3, right).

Remark $\mathbf{2}$ It may happen that $\left\langle\mathbf{x}^{-}\right\rangle$is empty, when $\mathbf{y}^{-}-\mathbf{b}^{-}>\mathbf{y}^{+}-\mathbf{b}^{+}$. To avoid useless calculus, an existence test is included in the algorithm SIVIA1. Moreover, we now have an enclosure for the constraints defined on $[\mathbf{x}]$, which is more accurate than the initial box $[\mathbf{x}]$, if $[\mathbf{x}]$ is small enough for the constraints to be approximately linear on it.

Eventually, from the box $[\mathbf{x}]$, the internal and external linearizations provide two polytopes included in $[\mathbf{x}]$, called $\left\langle\mathbf{x}^{-}\right\rangle$and $\left\langle\mathbf{x}^{+}\right\rangle$such that

$$
\left\langle\mathbf{x}^{-}\right\rangle \subset \mathbb{S}_{[\mathbf{x}]} \subset\left\langle\mathbf{x}^{+}\right\rangle
$$

where $\left\langle\mathbf{x}^{-}\right\rangle$is defined by $\left(\mathbf{A},\left[\mathbf{c}_{\mathbf{i}}\right]\right)$ and $\left\langle\mathbf{x}^{+}\right\rangle$is defined by $\left(\mathbf{A},\left[\mathbf{c}_{\mathbf{e}}\right]\right)$. The computation of the volumes of polytopes such as $\left\langle\mathbf{x}^{-}\right\rangle$and $\left\langle\mathbf{x}^{+}\right\rangle$is an important issue in mathematical programming [3]. Several free and downloadable softwares already exist. As an example, we propose here the lrs algorithm [2]. In SIVIA1 the exact computation of $\left\langle\mathbf{x}^{-}\right\rangle$and $\left\langle\mathbf{x}^{+}\right\rangle$ is performed on all the undetermined boxes $[\mathbf{x}]$ of $\mathcal{L}_{U}$.

\section{SIVIA1}

The main idea of the new algorithm is to obtain a greater accuracy in the description of $\mathbb{S}$ and of its volume while keeping the structure of SIVIA without increasing the number of bisections, with the reliable linearization tool of Section 2. Like SIVIA, SIVIA1 is a recursive algorithm designed to estimate a set $\mathbb{S}$, and especially its volume. The computed sets $\mathbb{S}^{-}$and $\mathbb{S}^{+}$are now hybrid: they are made of boxes $[\mathbf{x}]$ and polytopes $\langle\mathbf{x}\rangle$ whose computation is only performed in $\Delta \mathbb{S}$. Contrary to SIVIA, SIVIA1 does not furnish a regular paving of the bracketing sets. The tuning parameter $\varepsilon$ still directly controls the number of bisections. The smaller $\varepsilon$ is, the higher the number of bisections and the computational burden will be. This is partly compensated by the fact that the boxes of $\mathcal{L}_{U}$ will then be small, which diminishes the number of constraints to be linearized on the box and the smaller the boxes of $\mathcal{L}_{U}$ are 
too. This means that generally only one constraint $\mathbf{f}_{i}$ is to be linearized on the box, which facilitates the computation of the polytopes $\left\langle\mathbf{x}^{-}\right\rangle$and $\left\langle\mathrm{x}^{+}\right\rangle$. To focus on the performance of the reliable linearization itself, all the following results have been obtained on simple two-dimensional estimation problems. To compare the quality of the enclosures computed by SIVIA1 and SIVIA, we first study the bidimensional problem of Example 1. Figure 4 depicts the sets $\mathbb{S}^{-}$and $\mathbb{S}^{+}$defining the enclosure of $\mathbb{S}$. Let $\eta_{0}$ be the accuracy of $\mathbb{S}$ as estimated by computing the volume
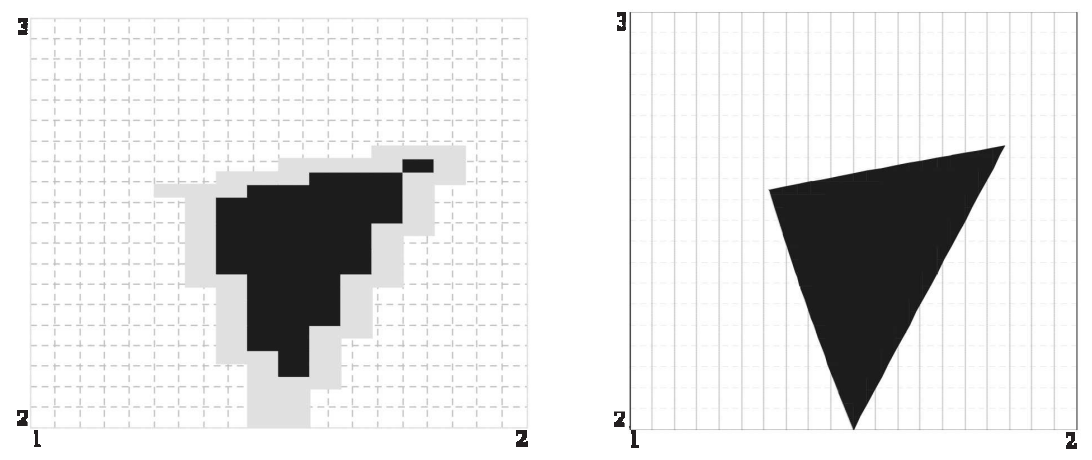

Figure 4 Left: SIVIA provides for $\varepsilon=0.05$, in 166 bisections in $0.05 \mathrm{sec}, \operatorname{Vol}(\mathbb{S})=$ [0.099;0.238]. Right: SIVIA1 provides for $\varepsilon=0.05$, in 166 bisections in $0.395 \mathrm{sec}$, $\operatorname{Vol}(\mathbb{S})=[0.163 ; 0.165]$.

of $\mathcal{L}_{U}$ generated by SIVIA, and $\eta_{1}$ be the accuracy of $\mathbb{S}$ computed by SIVIA1. Figure 5 confirms the gain obtained by using SIVIA1 instead of SIVIA. On Example 1, $\eta_{1}$ is a quadratic function of the computing time $t_{c}$ while $\eta_{0}$ is only linear as a function of $t_{c}$. We can now apply SIVIA1 to outlier detection.

Remark 3 We illustrated the utility of reliable linearization on an example where other methods would fail. For instance, interval constraint propagation would stop before reliable linearization. However on large boxes, ICP is more useful as it is faster and more accurate. A good idea is to combine these two techniques. For larger boxes, we use ICP, for small boxes the RL transform is sufficient, since on a small box the $f_{i}$ 's are nearly linear.

\section{SAFETY AND ROBUSTNESS}

As already explained, outliers may ruin estimation. An important task is then to design an efficient methodology to detect potential outliers. As we shall see, studying the influence of any particular datum on 


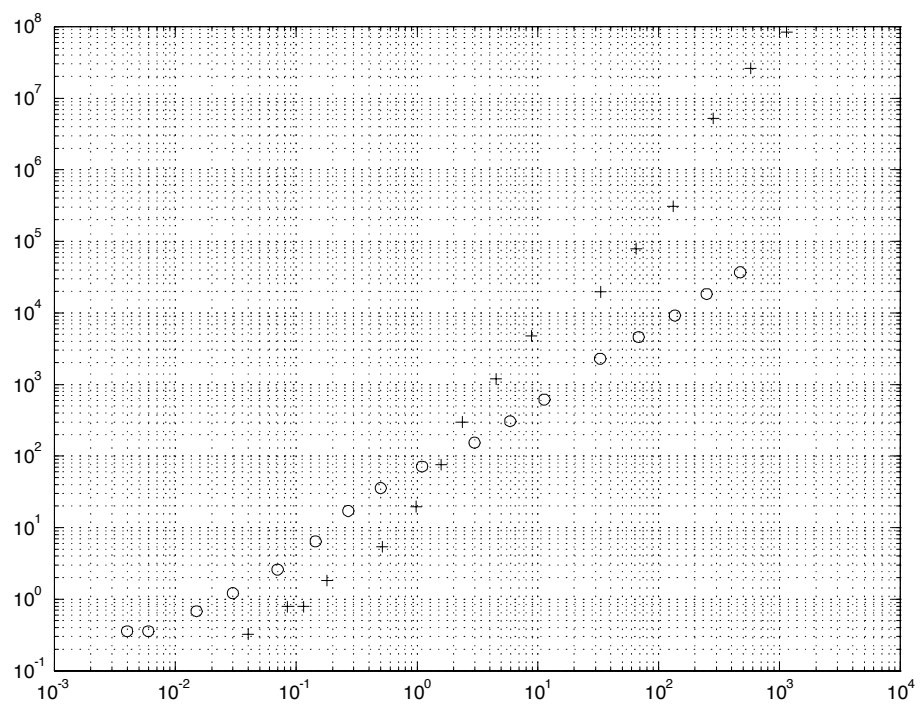

Figure $5 \eta_{1}(+)$ and $\eta_{0}(\mathrm{o})$ as functions of $t_{c}$.

the volume of $\mathbb{S}$ provides a way to check its coherence with the other data. Once a datum is identified as a potential outlier, it should be examined with particular care and may be discarded from $[\mathbf{y}]$, leading thus to an improvement of the robustness of the estimation, at the cost of an increase of the size of the feasible set. The concept of safety of datum will now be introduced for this purpose. To quantify the influence of the datum $\left[y_{i}\right]$ on the size of $\mathbb{S}$, we define its safety $\gamma_{i}[5]$ by the ratio

$$
\gamma_{i} \triangleq \frac{\operatorname{Vol}(\mathbb{S})}{\operatorname{Vol}\left(\mathbb{S}^{i}\right)}
$$

where $\mathbb{S}^{i}$ is the feasible parameter set obtained from $[\mathbf{y}]$ deprived of the $i$-th datum, i.e.

$$
\mathbb{S}^{i}=\left\{\mathbf{x} \in \mathbb{X} \mid f_{k}(\mathbf{x}) \in\left[y_{k}\right] \quad \forall k \in\{1, \ldots, i-1, i+1, \ldots, n\} .\right.
$$

The smaller $\gamma_{i}$ is, the less redundant is the $i$-th datum and the more careful we have to be with it. Note that

$$
\mathbb{S}=\cap_{i \in\{1, \ldots, n\}} \mathbb{S}^{i},
$$

so $\operatorname{Vol}(\mathbb{S}) \leq \operatorname{Vol}\left(\mathbb{S}^{i}\right) \quad \forall i \in\{1, \ldots, n\}$. The values taken by $\gamma_{i}$ can then be packed in three categories: 
- if $\gamma_{i}=0$, then $\mathbb{S}=\emptyset$ but $\mathbb{S}^{i} \neq \emptyset$ : taking into account the $i$-th datum reduces the nonempty set $\mathbb{S}^{i}$ into the empty set. While all the other data do not contradict each other, the $i$-th datum is very informative and likely to be an outlier.

- if $0<\gamma_{i}<1$ then $\mathbb{S} \neq \emptyset$ and $\operatorname{Vol}(\mathbb{S}) \leq \operatorname{Vol}\left(\mathbb{S}^{i}\right)$ : taking the $i$-th datum into account reduces the solution set: the closer the safety is to 1 , the safer it is to use it, as its information is consistent with that provided by other measurements.

- if $\gamma_{i}$ is not a number $(N a N)$, then $\mathbb{S}^{i}=\emptyset$, and there are more than one outlier. Specific techniques such as GOMNE [8] may then be employed.

The safety of data will thus allow us to determine which data points must be checked with particular attention. As $\mathbb{S}$ is bracketed between $\mathbb{S}^{-}$and $\mathbb{S}^{-}$, we obtain an interval estimate $\left[\gamma_{i}\right]$ of the safety of the $i$-th datum.

Example 2 We shall illustrate these principles assuming that $\mathbf{f}$ is from $\mathbb{R}^{2}$ to $\mathbb{R}^{5}$, and such that $\mathbf{f}(\mathbf{x})=x_{1} \sin \left(2 \pi x_{2} \mathbf{t}\right)$, where $\mathbf{t}$ is the vector of measurement time, the prior domain for $\mathbf{x}$ is $\mathbb{X}=[1 ; 10] \times[0 ; 1]$ and the uncertainty on each datum $y_{i}=x_{1}^{*} \sin \left(2 \pi x_{2}^{*} t_{i}\right)$ is assumed to be $\left[y_{i}\right]=$ $\left[y_{i}-0.5 ; y_{i}+0.5\right]$.

To compare the performances of SIVIA and SIVIA1, we first simulate the system with $\mathbf{x}^{*}=(5 ; 0.4)^{T}$, without noise, for $\mathbf{t}=(0 ; 2 ; 4 ; 6 ; 8)^{T}$. Table 1 gives the results of the computation of $\left[\gamma_{i}\right]$ for each datum $y_{i}$, for $\varepsilon=5.10^{-3}$ after 20934 bisections. Note that $\left[\gamma_{i}\right]=[0 ;+\infty]$ means that $0 \in\left[\operatorname{Vol}\left(\mathbb{S}^{i}\right)\right]$, so $\mathbb{S}^{i}$ may be empty or not described with enough accuracy. Thus some $y_{j}$ with $j \neq i$ may be an outlier, but the problem remains undetermined. We can at least determine that $y_{4}$ is the most informative datum, as its safety is much smaller than the others. Consider now the same problem but with $y_{4}=0$ (i.e. sensor failure at $t=t_{4}$, see Table 2). As all $\left[\gamma_{i}\right]_{1}$ are equal to $[0 ;+\infty]$ except $\left[\gamma_{3}\right]_{1}$ and $\left[\gamma_{4}\right]_{1}$ that are exactly $0, y_{3}$ and $y_{4}$ exclude each other, so at least one of them is an outlier. Assume now that there is a bounded additive noise $b$ such that $\left|b\left(t_{i}\right)\right| \leq 0.5$, for eight $t_{i}$ regularly spaced between 0.3 sec and 2.4 sec. Figure 6 depicts the data with their uncertainty interval, for the two cases considered (with or without an outlier at $t_{5}$ ), with their corresponding estimated safeties. When only one outlier occurs at $t=t_{5},\left[\gamma_{5}\right]_{1}$ is low (o): the potential outlier has been detected, which cannot be achieved by other outlier detection techniques, such as GOMNE as $S$ is non empty

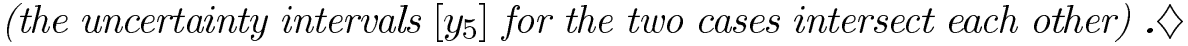


Table 1 Estimated safeties for each datum with $\operatorname{SIVIA}\left(\left[\gamma_{i}\right]_{0}\right)$ and SIVIA1 $\left(\left[\gamma_{i}\right]_{1}\right)$

\begin{tabular}{lccccc}
\hline$i$ & 1 & 2 & 3 & 4 & 5 \\
\hline$\left[\gamma_{i}\right]_{0}$ & {$[0 ;+\infty]$} & {$[0 ;+\infty]$} & {$[0 ;+\infty]$} & {$[0 ; 8.087]$} & {$[0 ;+\infty]$} \\
\hline$\left.\gamma_{i}\right]_{1}$ & {$[0.64 ; 0.87]$} & {$[0.62 ; 0.86]$} & {$[0.63 ; 0.86]$} & {$[0.38 ; 0.47]$} & {$[0.60 ; 0.72]$} \\
\hline
\end{tabular}

Table 2 Estimated safeties for each datum with $\operatorname{SIVIA}\left(\left[\gamma_{i}\right]_{0}\right)$ and $\operatorname{SIVIA1}\left(\left[\gamma_{i}\right]_{1}\right)$ with an outlier at $t=t_{4}$

\begin{tabular}{lccccc}
\hline$i$ & 1 & 2 & 3 & 4 & 5 \\
\hline$\left[\gamma_{i}\right]_{0}$ & {$[0 ;+\infty]$} & {$[0 ;+\infty]$} & {$[0 ;+\infty]$} & {$[0 ; 1.03]$} & {$[0 ;+\infty]$} \\
\hline$\left[\gamma_{i}\right]_{1}$ & {$[0 ;+\infty]$} & {$[0 ;+\infty]$} & {$[0 ; 0]$} & {$[0 ; 0]$} & {$[0 ;+\infty]$} \\
\hline
\end{tabular}

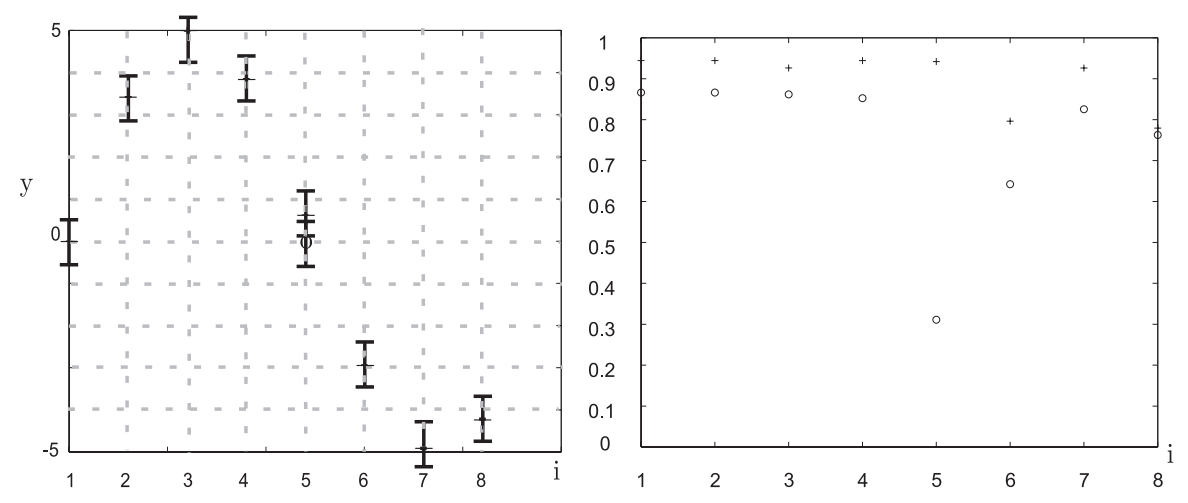

Figure 6 We consider two cases: no outlier occurred $(+)$, a sensor failure puts $y_{5}$ to zero (o). Left: the simulated model with the uncertainties. Right: the estimated safeties computed with SIVIA1 with a great acuracy : as the value of $\left[\gamma_{5}\right]_{1}$ is low, $y_{5}$ has to be checked carefully.

\section{CONCLUSIONS}

Parameter identification in a bounded-error context can be defined as a set-inversion problem. SIVIA is a branch-and-bound algorithm for solving this problem that estimates the set of all parameters consistent with the data given the bounds on the error by bracketing it between two unions of non-overlapping boxes. Unfortunately, an accurate computation of the volume of this set requires a very accurate estimation of the 
enclosure, only performed by SIVIA through many bisections. In order to limit the computational burden we have proposed a new tool based on the computation of internal and external polytopes in the uncertainty layer. These polytopes are computed via a reliable linearization. The performances of the resulting algorithm SIVIA1 show that, even if the problem remains NP-hard, it becomes possible to limit the violence of the numerical explosion of complexity. The importance of an accurate computation of volumes is illustrated by the use of the safety criterion to characterize measurements. This quantitative criterion based on volume computations aims at qualifying the less redundant data, i.e. the ones that are the most dangerous if they turn out to be outliers.

\section{References}

[1] E. R. Hansen. Bounding the solution of interval linear equations. SIAM Journal of Numerical Analysis, 29(5):1493-1503, 1992.

[2] http://elib.zib.de/pub/packages/mathprog/polyth/.

[3] http:///math.washington.edu/ ${ }^{2}$ hillman/PUB/Volume.

[4] L. Jaulin. Interval constraint propagation with application to bounded-error estimation. Automatica, 36:1547-1552, 2000.

[5] L. Jaulin, J. L. Godet, E. Walter, A. Elliasmine, and Y. Leduff. Light scattering data analysis via set inversion. Journal of Physics A: Mathematical and General, pages 7733-7738, 1997.

[6] L. Jaulin, M. Kieffer, I. Braems, and E. Walter. Guaranteed nonlinear estimation using constraint propagation on sets. International Journal of Control (accepted for publication), 2000.

[7] L. Jaulin and E. Walter. Set inversion via interval analysis for nonlinear bounded-error estimation. Automatica, 29(4):1053-1064, 1993.

[8] L. Jaulin, E. Walter, and O. Didrit. Guaranteed robust nonlinear parameter bounding. In Proc. CESA'96 IMACS Multiconference (Symposium on Modelling, Analysis and Simulation), pages 11561161, Lille, July 9-12, 1996.

[9] R. E. Moore. Methods and Applications of Interval Analysis. SIAM Publ., Philadelphia, 1979.

[10] R. E. Moore. Parameter sets for bounded-error data. Mathematics and Computers in Simulation, 34:113-119, 1992. 